\title{
Dietary integration with galactose, Coenzyme Q and reduced glutathione healed low back pain: A case report
}

\author{
Isabella Panfoli* and Daniela Calzia \\ Dipartimento di Farmacia, Laboratorio di Biochimica, Università di Genova, Italy
}

\begin{abstract}
This paper deals with the case of a 79-year-old female patient presenting chronic low back pain secondary to total knee replacement 6 months before, to whom physical therapy had negligible effect. Score 10 on the leeds assessment of neuropathic symptoms and signs pain scale ruled out neurologic causes. A plain X-ray scan showed widespread signs of spondylosis, slight anteposition of L3, multiple lumbar discopathies, but no disk herniation. Physical examination, and assessment of Numeric Pain Rating Scale, were conducted during her initial visit, after six weeks and at 8-month follow-up. Treatment consisted of daily integration with two sub-tongue patented tablet containing: galactose $(500 \mathrm{mg})$, reduced glutathione $(50 \mathrm{mg})$ and Coenzyme Q10 (50 mg) after evening meal. After 6 weeks of supplementation, back pain had disappeared, and all outcome measures were improvement.

Numeric Pain Rating and Oswestry Disability Index decreased from 7/10 to 3/10 and from 54\% to15\% respectively and Pain Self -Efficacy Questionnaire from the value $18 / 60$ to $49 / 60$. At 8 -month follow-up, all outcome measures continued to be improved and back pain did not occur again, even though the patient presently suffers from a severe gonalgia limiting her movements.
\end{abstract}

\section{Introduction}

Low Back Pain (LBP) is a common condition of pain in the lumbar region [1], extended between the lower costal margin and the intergluteal cleft, often accompanied by muscular rigidity. Normally, pain irradiates to the lower limb. More than $70 \%$ adults will suffer from it in their life, significantly affecting the quality of life. The socio-economic weight of invalidity for chronic LBP is increasing exponentially in industrialized countries. The most common causes of back pain are mechanical or due to lumbar strain, while degenerative processes of disks and disk herniation or spondylolisthesis are less common. Non-mechanical spinal conditions (neoplasia, infection, arthritis, visceral or pelvic organ disease, aortic aneurysm) account for less than 1\%. Prevalence of serious spinal pathology is low [2]. Strategies exist to screen for clinical pathologies that can mimic LBP [3]

LBP can be acute or chronic. In the first case, the maximum duration of the painful episode must not exceed 12 weeks. A duration of painful symptomatology longer than 3 month classifies LPB as chronic LBP. Not all back pain requires imaging; LBP lasting more than 3 months is considered chronic and should be imaged. If neurological signs are associated imaging should be done best by Magnetic Resonance Imaging [4] but lumbar spine standard radiographs are the first line intervention, due to its low cost. Plain film allows visualization of bony structures, although soft tissue is not well visualized. Evidence for which physical therapy is better in those with chronic LBP and multiple health conditions is lacking.

This case report describes a non-conventional management of a patient with chronic LBP and multiple health conditions.

\section{Materials and Methods}

\section{Presenting Concerns}

Patient Profile: Age: 79 Height: $155 \mathrm{~cm}$ Weight: $75 \mathrm{Kg}$. BMI 30. Mean BP 130/82 mmHg. Condition: chronic low back pain. Social
History: Former high-school teacher woman, normally active and enjoying walking regularly.

\section{Clinical Findings}

General health: no family history of lower back pain, diabetes, epilepsy. Past Medical History: hypothyroidism secondary to Hashimoto thyroiditis since 2001; various non-specified allergies. Prescribed: Levothyroxine $50 \mathrm{mcg}$ (daily); Surgical History: excision of colon adenomatous malignant polyp, 2002, followed by systemic adjuvant chemotherapy with fluorouracil.

\section{Results}

Patient presented with 8-months history of persistent dull LBP, with pain radiating to the left leg from buttock to heel. Walking for long periods and housework exacerbated pain. Pain-related sleep disruption and progressive limitation to walk were the primary activity restrictions. The patient had undergone complete prosthetic replacement of the right knee 5 months before but was otherwise well.

Active movements (Patient performed) were restricted and painful in all planes. There was local tenderness at the palpation of distal L3 joint of the lumbar spine, to the left side of the back, with muscular spasm. No spinal deformity. Straight leg raise test [5] was normal, excluding as

*Correspondence to: Isabella Panfoli, $\mathrm{MD}, \mathrm{PhD}$-Biochemist University of Genova-DIFARV.le Benedetto XV, 316132 Genova, Italy, Tel: + 390103537397 ; Fax: +390103538153; E-mail: panfoli@difar.unige.it

Key words: coenzyme Q10, galactose, low back pain, oxidative phosphorylation, neuroprotection

Received: September 28, 2018; Accepted: October 09, 2018; Published: October 12,2018 
an underlying herniated disk. Neurological sensory and motor testing was normal and symmetrical. Ankle clonus and Babinski reflex were not present. Absence of weight loss, night-time fever/ sweats, fatigue, pins and needles/ numbness in groin region, alterations to bladder and bowel function, allowed to exclude serious pathology [4] even though the patient had a history of cancer. There was evidence of significant sciatic nerve irritation. Blood and urine analyses were unremarkable. Due to the severity of symptoms but considering her allergy to main Nonsteroidal anti-inflammatory drugs her general practitioner had prescribed paracetamol ( $1 \mathrm{~g} \times 2 /$ daily) with little effect. Symptom persistence led to suspect the involvement of the intervertebral discs, causing compression to the sciatic nerve root and leg symptoms. As patient did not improve after 3 months of pharmacologic treatment, clinician considered addition of nonpharmacologic therapy namely spinal manipulation and rehabilitation, exercise therapy. It was recommended referral to physical therapy management with spinal manipulation therapy, with a physiotherapist, but this did not improve outcomes 6 months after symptom onset. Her pain has improved but not resolved. The patient was then referred to a local orthopedic consultant, specialist in spinal injury in Genoa. As LBP lasted more than 3 months it was considered chronic and was imaged. The clinician prescribed an X-Ray scan of the spine. Scan identified that the disc contents had not leaked out even though there were widespread signs of spondylosis with marginal osteophytosis, slight anteroposition of L3. The working diagnosis was a mild L3 dysfunction with involvement of ischiatic nerve irritation. Based on this results, surgical procedure was not indicated. It was decided to follow the patient conservatively with follow-up in 6 weeks the physical exam mimicked what one would expect of a patient with a benign musculoskeletal condition.

\section{Physical examination}

\section{X-Ray Scan}

As reported in Figure 1, the most pertinent findings at the imaging $\mathrm{X}$-ray scan were: double curved dorso-lumbar scoliosis. Partial
A

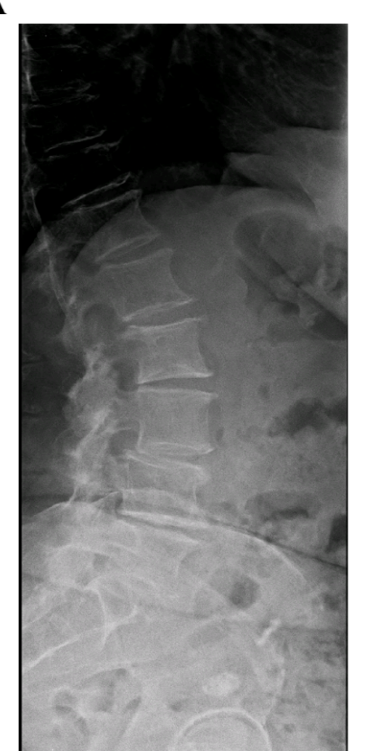

B

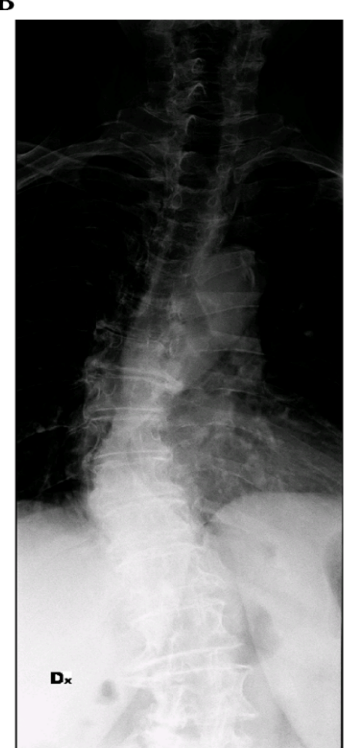

Figure 1. Anteroposterior and lateral X-ray scans of spine prior nutritional treatment Panel A Anteroposterior-view plain X-ray scan of the lumbar spine showing signs of spondylosis with marginal osteophytosis, slight anteroposition of L3.

Panel B Latero-lateral-view of plain X-ray scan of the lumbar spine in extension position showing scoliotic rotation of metamers. rotation of metamers. Widespread signs of spondylosis with marginal osteophytic protrusions. Slight anteroposition of L3. Multiple lumbar discopathies. Diffuse degenerative discs in the middle and lower tract of the dorsal spine; osteoporotic spine, but no disk herniation.

\section{Diagnostic Focus and Assessment}

Physical examination, and assessment of Numeric Pain Rating Scale (NPRS), Oswestry Disability Index (ODI), and Pain Self -Efficacy Questionnaire (PSEQ), were conducted during her initial visit, after six weeks and at 8-month follow-up.

\section{Therapeutic Focus and Assessment and Timeline}

Treatment was designed to allow neuroprotection, considering that pain mechanism was essentially irritation of ischiatic nerve. The focus of the intervention was neuro-protective for the ischiatic nerve, with an oral daily integration with two subtongue patented tablet containing: galactose $(500 \mathrm{mg})$, reduced glutathione $(50 \mathrm{mg})$ and Coenzyme Q10 $(50 \mathrm{mg})$ after evening meal. 6 weeks later, the patient reported dramatic change in her condition, as symptoms had disappeared. Treatment consisted of daily integration.

\section{Follow-up and Outcomes}

Outcome measures included the NPS, ODI and PSEQ value. A score 10 on the leeds assessment of neuropathic symptoms and signs pain scale allowed to rule out neuropathic mechanisms contributing to the patient's pain. Therefore, Magnetic Resonance Imaging was not recommended. Six weeks after the beginning of oral integration, the patient's NPRS,ODI, and PSEQ scores improved by more than $80 \%$ without any medication (Table 1)

\section{Discussion}

Authors report herein a patient presenting with low back pain. Normally LBP is caused by musculoskeletal reasons, and does not require particularly any intervention or treatment, but often damaged. Intervertebral discs are the cause of pain. In this case, pain can become chronic and the management of a patient with a chronic LBP and sometimes multiple health conditions can pose problems as far the clinical decision. Spinal manipulation can sometimes be effective, although it is unclear to what extent [6].

National guidelines are not in accordance as for the recommendation of manipulation or physical therapy [7], which is commonly adopted. Patients with low back pain must first be into one of three broad categories with accurate anamnestic and physical examination: nonspecific LBP, back pain potentially associated with radiculopathy or spinal stenosis, or back pain potentially associated with a sinister (when severe or progressive neurologic deficits) are present or other specific spinal cause. Our case was placed in the second category. Accordingly, diagnostic imaging and testing was limited. Paracetamol is a first-line therapy for managing acute low back pain, as its efficacy is well established. Relief, in the case of our patient even though utilizing regular use of appropriate doses ( $1 \mathrm{~g}$ twice a day) only provided moderate relief. Patient did not utilize Nonsteroidal anti-inflammatory drugs, due to her allergy, even though their efficacy in low back pain is limited. Therefore, the patient was not envisaging any efficacious treatment. In fact, neuroprotection is considered not applicable, especially in these mild cases. In this context, we considered both our previous biochemical data and clinical results. The former showed that the mitochondrial $\mathrm{F}_{0} \mathrm{~F}_{1}$ - ATP synthase, and the respiratory complexes are expressed in myelin [8-13] which together with the axonal mitochondria, would supply the axoplasm with aerobically 
Table 1. Assessment of pain and disability

\begin{tabular}{|c|c|c|c|}
\hline & Baseline & 6 weeks & 8 months \\
\hline NRS & 7 & 3 & 1 \\
\hline ODI & 38 & 15 & 13 \\
\hline PSEQ & 18 & 49 & 51 \\
\hline
\end{tabular}

Table reports the assessment of pain and disability, before (baseline) and at the end ( 6 weeks) of the nutritional treatment and at a follow up after 8 months. NPRS Numerical rating Scale (range 0-10) ODI Oswestry Disability Index (range 0-100) and PSEQ Pain Self Efficacy Questionnarie (range 0-60) were evaluated.

synthesized ATP, through connexons [14]. The latter is a case report which showed that dietary neuroprotecting daily integration with galactose $(3 \mathrm{~g})$ and Coenzyme Q10 (100 mg) in a woman with a brain Magnetic Resonance Imaging showing periventricular and callosal multiple lesions suggestive of a demyelinating nature, allowed to delay the diagnosis of Multiple Sclerosis after three follow-up within 18 months now, regardless of the fact that patient's condition was considered strongly suggestive of early Multiple Sclerosis.

In fact, D-galactose would play a role as a bioenergetics substrate for the extra-mitochondrial ATP synthesis and oxygen consumption in myelin. In fact, demyelinated axons eventually degenerate. It was supposed that D-galactose can become the substrate of hexose6-phosphate dehydrogenase, whose functional expression was shown in isolated myelin which would be functionally associated to respiratory Complex I, expressed in myelin [15]. Hexose-6-phosphate dehydrogenase is a microsomal enzyme expressed in the endoplasmic reticulum, which has a particularly favorable $\mathrm{K}_{\mathrm{M}}$ for galactose.

Galactose can enter the nerve cell across GLUT3, an hexose transporter which is not insulin-dependent [16]. Galactose can increment the oxidative burst in the cell. A beneficial effect of oral galactose was reported in preventing the development of the cognitive deficits in the streptozotocin-induced rat model of sporadic Alzheimer's disease [17]. Along this view is the association with coenzyme Q10 for its function in funneling electrons through the electron transfer chain. It is also important to acknowledge that with the cited dietary integration the patient did not develop a worsening of her condition. In this respect, the integration with galactose/Coenzyme Q10, an approach intended support the myelin bioenergetics, may have exerted a neuroprotective effect, thereby reducing further insults to the white matter, thereby allowing the nerve to heal.

Notably, supplementation with galactose is recommendable, in the early stages of damage to nerves in order to support the other therapies in helping myelin to aerobically support the axon with chemical energy, which can accelerate healing.

\section{Declaration of Interest}

No potential conflict of interest was reported by the authors

\section{Funding}

This research did not receive any specific grant from funding agencies in the public, commercial, or not-for-profit sectors.

\section{References}

1. Chou R, Qaseem A, Snow V, Casey D, Cross JT, et al. (2007) Clinical Efficacy Assessment Subcommittee of the American College of Physicians, American College of Physicians, American Pain Society Low Back Pain Guidelines Panel. Diagnosis and treatment of low back pain: a joint clinical practice guideline from the American College of Physicians and the American Pain Society. Ann Intern Med 147: 478-91. [Crossref]

2. Henschke N, Maher CG, Refshauge KM, Herbert RD, Cumming RG, et al. (2009) Prevalence of and screening for serious spinal pathology in patients presenting to primary care settings with acute low back pain. Arthritis Rheum 60: 3072-3080. [Crossref]

3. Deyo RA, Diehl AK Lumbar spine films in primary care: current use and effects of selective ordering criteria. J Gen Intern Med 1: 20-25. [Crossref]

4. Ross MD, Boissonnault WG (2010) Red Flags: To Screen or Not to Screen? J Orthop Sport Phys Ther 40: 682-684. [Crossref]

5. Manusov EG (2012) Evaluation and Diagnosis of Low Back Pain. Prim Care Clin Off Pract 39: 471-479. [Crossref]

6. Walker BF, French SD, Grant W, Green S (2011) A Cochrane review of combined chiropractic interventions for low-back pain. Spine (Phila Pa 1976) 36: 230-242.

7. Qaseem A, Wilt TJ, McLean RM, Forciea MA (2017) Clinical Guidelines Committee of the American College of Physicians. Noninvasive Treatments for Acute, Subacute, and Chronic Low Back Pain: A Clinical Practice Guideline From the American College of Physicians. Ann Intern Med 166: 514-530.

8. Ravera S, Panfoli I, Calzia D, Aluigi MG, Bianchini P, et al. (2009) Evidence for aerobic ATP synthesis in isolated myelin vesicles. Int J Biochem Cell Biol 41: 15811591. [Crossref]

9. Ravera S, Panfoli I, Aluigi MG, Calzia D, Morelli A (2011) Characterization of Myelin Sheath $\mathrm{F}(\mathrm{o}) \mathrm{F}(1)$-ATP synthase and its regulation by $\mathrm{IF}(1)$. Cell Biochem Biophys 59: 63-70. [Crossref]

10. Ravera S, Nobbio L, Visigalli D, Bartolucci M, Calzia D, et al. (2013) Oxydative phosphorylation in sciatic nerve myelin and its impairment in a model of dysmyelinating peripheral neuropathy. $J$ Neurochem 126: 82-92.

11. Ravera S, Bartolucci M, Calzia D, Aluigi MG, Ramoino P, et al. (2013) Tricarboxylic acid cycle-sustained oxidative phosphorylation in isolated myelin vesicles. Biochimie 95: 1991-1998.

12. Bartolucci M, Ramoino P, Calzia D, Traverso C, Panfoli I. RS. Oxydative Metabolism in Optic Nerve Myelin: New Perspectives in Hereditary Optic Neuropathies. Clin J Ophthalmol.

13. Morelli A, Ravera S, Panfoli I (2011) Hypothesis of an Energetic Function for Myelin Cell Biochem Biophys 61: 179-187. [Crossref]

14. Ravera S, Bartolucci M, Adriano E, Garbati P, Ferrando S, et al. (2016) Support of Nerve Conduction by Respiring Myelin Sheath: Role of Connexons. Mol Neurobiol 53: 2468-2479. [Crossref]

15. Ravera S, Bartolucci M, Calzia D, Morelli A, Panfoli I (2015) Galactose and Hexose 6-Phosphate Dehydrogenase Support the Myelin Metabolic Role. PARIPEX-Indian J Res IV: $397-400$.

16. Olson AL, Pessin JE (1996) Structure, function, and regulation of the mammalian facilitative glucose transporter gene family. Annu Rev Nutr 16: 235-56.

17. Salkovic-Petrisic M, Osmanovic-Barilar J, Knezovic A, Hoyer S, Mosetter K, et al (2014) Long-term oral galactose treatment prevents cognitive deficits in male Wistar rats treated intracerebroventricularly with streptozotocin. Neuropharmacology 77 : 68-80. [Crossref]

Copyright: (C2018 Wilson JR. This is an open-access article distributed under the terms of the Creative Commons Attribution License, which permits unrestricted use, distribution, and reproduction in any medium, provided the original author and source are credited. 